\title{
Philosophiques
}

\section{Philosophie et marketing : Sartre à Montréal, mars 1946}

\section{Yvan Cloutier}

Volume 15, numéro 1, printemps 1988

URI : https://id.erudit.org/iderudit/027041ar

DOI : https://doi.org/10.7202/027041ar

Aller au sommaire du numéro

Éditeur(s)

Société de philosophie du Québec

ISSN

0316-2923 (imprimé)

1492-1391 (numérique)

Découvrir la revue

Citer cet article

Cloutier, Y. (1988). Philosophie et marketing : Sartre à Montréal, mars 1946. Philosophiques, 15(1), 169-190. https://doi.org/10.7202/027041ar

\section{Résumé de l'article}

À quoi attribuer le succès médiatique de Sartre à Montréal en mars 1946 ? Quelles furent les conditions de la réception de cette philosophie dans la culture québécoise ? Cette étude montre que le succès médiatique de Sartre est rendu possible principalement par l'action de journalistes et de critiques littéraires qui exploitèrent les thématiques de la philosophie sartrienne en vue d'ouvrir le climat intellectuel québécois et d'accroître leur pouvoir dans le champ intellectuel. d'utilisation que vous pouvez consulter en ligne.

https://apropos.erudit.org/fr/usagers/politique-dutilisation/ 


\title{
PHILOSOPHIE ET MARKETING : SARTRE A MONTRÉAL, MARS $1946^{\circ}$
}

\author{
par Yvan Cloutier
}

\begin{abstract}
RÉSUMÉ À quoi attribuer le succès médiatique de Sartre à Montréal en mars 1946 ? Quelles furent les conditions de la réception de cette philosophie dans la culture québécoise? Cette étude montre que le succès médiatique de Sartre est rendu possible principalement par l'action de journalistes et de critiques littéraires qui exploitèrent les thématiques de la philosophie sartrienne en vue d'ouvrir le climat intellectuel québécois et d'accroitre leur pouvoir dans le champ intellectuel.
\end{abstract}

ABSTRACT. What contributed to Sartre's Montreal media success of March 1946? What conditionned the enthusiastic reception of Sartre's philosophy? This study shows that journalists and literary critics were largely responsible for the French philosopher's media success. They used Sartre's philosophy for the purpose of expanding the cultural climat while extending their own power base within Quebec's intellectual life.

Les philosophies empruntent des canaux institutionnels (revues, cours, séminaires, clubs, conférences, etc.) qui, dans nombre de cas, en conditionnent la réception au même titre que leur qualité intellectuelle intrinsèque; c'est ainsi que l'on peut comprendre l'irruption de modes philosophiques ${ }^{2}$ ou d'engouement à l'endroit de philosophies comme le marxisme au Québec ${ }^{3}$ dans les années

1. Ce texte est une version remaniée d'une communication présentée lors du Congrès de l'A.C.F.A.S., Montréal, le 15 mai 1986. Je remercie Roland Houde et Jacques Michon pour leur collaboration à titre d'experts et de lecteurs.

2. Voir: Yvan Cloutier, Des «modes» philosophiques : le cas Sartre. Communication prononcée au Congrès de l'Association Canadienne de Philosophie, 29 mai 1985, (texte photocopié), 12 pages.

3. En particulier l'UQAM. 
1960 et aux États-Unis dans les années $1970^{4}$. Si les philosophes consomment des «Macphilosophies » ${ }^{5}$, qu'en est-il des nonprofessionnels de la philosophie? Peut-on vendre de la philosophie comme on vend des «Big-macs»? Peut-on mener une opération marketing de la philosophie? Perçue comme une discipline très peu accessible à cause des capacités intellectuelles qu'elle exige de ses praticiens, la philosophie semblerait devoir échapper aux contraintes du monde médiatique.

Le cas Sartre illustre bien cette " diffusivité » d'une philosophie. Non satisfait de son succès dans le monde littéraire auprès des experts et dans le monde philosophique surtout auprès des professeurs de lycée, Sartre vise une audience élargie ${ }^{6}$; Anna Boschetti a montré comment la vulgarisation du projet s'inscrit dans la stratégie sartrienne pour occuper une place prépondérante dans le champ intellectuel en profitant des changements structurels (scolarisation, importance de la critique littéraire dans les journaux, élargissement du public) et conjoncturels (crise économique, guerre, développement de l'édition, etc.). Les succès d'édition, les salles remplies, les témoignages sur la «mode existentialiste», voire même un sondage auprès des Français ${ }^{7}$ attestent cette très large diffusion de l'existentialisme sartrien.

Mais à quoi faut-il attribuer cette très large diffusion du sartrisme? Deux types d'explication s'opposent : la première y voit un succès médiatique et met l'accent sur les appareils de diffusion culturelle, alors que la deuxième utilise une sociologie des contenus et insiste sur le caractère «organique » d'une conception du monde. Ces deux explications ont été proposées pour expliquer la vogue pour Sartre de mars 1946 à Montréal ${ }^{8}$.

4. Voir : Richard W. Miller, «Marx in Analytic Philosophy: The Story of a Rebirth ». Social Science Quaterly, vol. 64, no 4 (déc. 1983), p. 846-861.

5. Cette notion n'est nullement péjorative, $j$ 'y inclus certes l'existentialisme, le thomisme, la phénoménologie, le marxisme mais aussi ces approches « rigoureuses » que sont le structuralisme et la philosophie analytique.

6. Anna BoschetT1, Sartre et «Les Temps Modernes», Paris, Les Éditions de Minuit, 1985 , p. 76.

7. B.P. O'DonOHOE, «Connaissez-vous Sartre? 》 Obliques (numéro spécial consacré à Sartre), no 18-19, Paris, 1979, p. 335-341.

8. Ce texte s'inscrit dans la foulée du travail du Professeur Roland Houde : «Sartre ici bibliographie anatomique (préliminaire) », La petite revue de philosophie, vol. 2, n $\mathrm{n}^{\circ} \mathrm{I}$ (automne 1980), p. 137-161 et d'un dossier composé de notes autographes et de documents, daté 19-30 novembre 1981 et intitulé Sartre au Québec (1939-1970), 65 p. 
A près avoir décrit la réception de Sartre à Montréal en mars 1946, je montrerai que ce succès médiatique est rendu possible par l'action de critiques littéraires qui exploiteront la "traductibilité » des thématiques de la philosophie sartrienne en vue d'ouvrir le climat intellectuel et d'améliorer leur propre pouvoir dans le champ intellectuel québécois. Dès lors, «contenus » et médias renvoient l'un à l'autre.

\section{SUCCÈS MÉDIATIQUE}

Mars 1946: point culminant d'une vogue Sartre à Montréal. Précédée du succès de Huis Clos et d'une polémique autour de l'existentialisme sartrien, la venue de Sartre à Montréal le 10 mars 1946 donne lieu à un succès médiatique sans précédent dans l'histoire de la philosophie au Québec au $\mathrm{XX}^{\mathrm{e}}$ siècle.

Les journaux (La Presse, Le Canada, Le Devoir, La Patrie, Montréal-Matin) annoncèrent largement (entre le 4 et le 9 mars) la conférence de Sartre du 10 mars à la Société d'étude et de conférences ; la conférence était intitulée «La littérature française de 1914 à 1945; la littérature clandestine ». Les 6, 7 et 8 mars, le Canada français publie le texte d'une conférence de l'éditeur Lucien Parizeau au Sénat de la Jeunesse sur «L'engagement de l'écrivain» dans lequel il est largement question de Sartre.

Cette conférence de Sartre à l'hôtel Windsor devant plus de 600 personnes est enregistrée par Radio-Canada. Lundi le 11 mars, Sartre figure à la une du Canada (photographie)! Le Devoir, la Patrie, le Quartier Latin et la Presse, publient un compte rendu de la conférence de presse donnée par Sartre. Quelques-uns de ces journaux iront jusqu’à résumer la conférence de Sartre à la S.E.C. (La Patrie, Notre Temps, Revue Populaire).

Le Canada publie en page 4 de son édition du 12 mars une caricature de Sartre signée par Robert LaPalme?. Les 14 et 15 mars, les Montréalais lisent dans Le Canada, La Presse, MontréalMatin, La Patrie et Le Devoir un compte rendu de la conférence du

9. Cette caricature sera reprise dans le Sartre par lui-même de Francis Jeanson (p. 82) sans mention de la source québécoise. 
critique et philosophe Guy Sylvestre au Cercle Universitaire où l'auteur propose son explication de la vogue Sartre ${ }^{10}$.

L'examen des mentions du nom Sartre dans Le Quartier Latin, Le Devoir, Notre Temps, Le Canada et La Presse ${ }^{11}$ indique que la présence de Sartre dans les médias augmente brusquement en janvier pour atteindre rapidement son sommet en mars, refait surface occasionnellement jusqu'en janvier 1948, et finit par s'estomper (il y a au moins une mention en 51 mois sur 60 , entre 1949 et 1954).

Le grand nombre de médias montréalais impliqués, leurs tirages élevés ${ }^{12}$ et la concentration des messages en un court laps de temps contribuèrent à créer un «hit», ce type d'attention privilégiée que cherche tout publicitaire ${ }^{13}$. «L'existentialisme est à la mode: tout le monde en parle, il est presque impossible d'ouvrir un journal ou une revue, d'entrer dans un salon ou un restaurant, sans rencontrer ou entendre le nom de Sartre »; voilà comment Guy Sylvestre décrivait ce succès médiatique qu'il attribuait à des « chroniqueurs en mal de copie et des caricaturistes en mal de sujet [qui] ont ridiculisé l'existentialisme et ont ainsi réussi à donner à ce mouvement philosophique une popularité universelle ${ }^{14}$.

10. Le texte de la conférence sera publié dans La Nouvelle Relève d'avril.

11. Voici la compilation des mentions implicites et explicites : janvier (13), février (10), mars (35), avril (2), mai (9), juin (3), juillet (7), août (7), septembre (6), octobre (4), novembre (7), décembre (8) ; alors que la moyenne de mention est de 9.25 en 1946 , elle sera de 3.33 en 1947 et de 2.17 en 1948. Je n'ai trouvé que huit (8) mentions en 1945 mars (3), octobre (4), et décembre (1).

12. Les tirages en 1940 : La Presse (147,074), Le Petit Journal (90,095), La Patrie $(29,000)$, Le Devoir (20,000), Le Jour (10,000), Le Canada (13,500) et Montréal-Matin (30,000). D'après André Beaulieu, Jean Hamelin, Les journaux du Québec de 1764 à 1964, Québec, Presses de l'Université Laval, 1965 et Jean-Paul DE LaGrave, Liberté et servitude de l'information au Québec confédéré (1867-1967), Montréal, Éditions de Lagrave, 1978

13. Deux faits cocasses témoignent d'un effet-Sartre dans le langage médiatique; dans le premier, le chroniqueur de cinéma de La Presse écrivait dans un article du 16 mars 1946 et intitulé « Un direct à l'absurde » : “ Faudrait-il parler d'existentialisme au sujet des vedettes de cinéma?... Bien sûr, en autant que la théorie de Jean-Paul Sartre indique jusqu'à quel point il existe des choses absurdes de par le monde du cinéma. Quoi de plus absurde, en effer, que le système de vedettes ? " d dans le deuxième cas, un éditeur publie dans Le Devoir du 16 mars 1946 une annonce pour le livre La Quête de l'Existence d'Edmond LABELLE publié en 1944 et sans rapport avec l'existentialisme sartrien; on y lit: «L'existentialisme est à la page! Pour en avoir une notion exacte, lisez... 》.

14. Guy Syivestre, “ Les personnes du drame », Le Droit, 23 mars 1946, p. 2. 
Mais à quoi attribuer ce succès de Sartre dans les médias et l'effet-mode ou engouement que l'on a attribués aux médias? Qu'est-ce qui fait que Sartre était un «bon vendeur » en 1946 et que Borduas ne pouvait pas l'être en 1948 ? Ce succès dans les médias est-il un succès de médias, imputable à la seule action des médias? Ou doit-on prendre en considération les « horizons d'attente» des divers groupes en lutte dans le champ intellectuel? Les intellectuels de ces groupes opèrent à l'intérieur de leur conception (implicite et explicite) du monde et de la lecture qu'ils font des attentes des groupes qu'ils veulent diriger. Ainsi avant même d'être diffusée, une conception du monde doit répondre aux attentes des diffuseurs et à la lecture qu'ils font des attentes des lecteurs.

\section{UN SUCCÈS DE MÉDIAS}

\subsection{Un «ballon» médiatique?}

Dans son analyse de la réception de Sartre aux États-Unis en 1946, Annie Cohen-Solal ${ }^{15}$ accorde beaucoup d'importance aux médias ; les «médias français avaient mis la fusée sur orbite; les médias américains allaient, selon certaines de leurs méthodes, le plonger tout vif dans le bain du «star system» ${ }^{16}$. Les grands journaux et les revues «diffusent » Sartre; Time Magazine lui consacre un important article dans une sous-section «Existentialism » de la section «Europe » de sa rubrique «Foreing News » ${ }^{17}$; suivront le New York Post, le New Yorker, la Partisan Review et le Harper's Bazaar.

Annie Cohen-Solal décrit aussi le rôle clef de Dolorès Vanetti dans cette promotion de Sartre et le rôle de « relais » que joueront plusieurs universitaires français installés aux États-Unis, notamment Jean-Albert Bédé (Columbia University), Henri Peyre (Yale University) ${ }^{18}$ et Jean Seznec (Harvard University). Grâce à une stratégie bien orchestrée, "bientôt, écrit Cohen-Solal, à New

15. Sartre, Paris, Gallimard, 1985,729 p.

16. Ibidem, p. 357 ; voir : 362,364 .

17. Time Magazine, vol. 47, $\mathrm{n}^{\circ} 4$ (January 26), 1946, pp. 20-21.

18. Selon Cohen-Solal, Peyre était "l'homme clef de la vie litréraire et universitaire française aux U.S.A.» (p. 359). 
York, chacun eut son Sartre, les universitaires, philosophes ou littéraires, les journalistes, les dramaturges, les politiques, les romanciers... ${ }^{19}$.

Contrairement aux Américains, les universitaires montréalais (sauf Ceslas Forest, o.p.) ne jouèrent aucun rôle dans la réception de Sartre. La partie se joua dans les médias où concoururent un phénomène de mondainisation et le pouvoir nouveau et croissant de la critique littéraire dans le champ culturel québécois. L'éditeur Lucien Parizeau ${ }^{20}$ joua un rôle important et les relais furent la Société d'étude et de conférences et des journalistes auvrant dans des journaux libéraux.

\subsection{Mondainisation et médias}

André Langevin, alors responsable des pages littéraires du Devoir d'où partira la réaction contre Sartre, explique la montée rapide de Sartre par la mondainisation; «Dire, écrit-il, que M. Sartre est venu l'an dernier et que son passage a été inaperçu! La roue tourne et ses pointes ne sont point toutes semblables... Du vent dans un ballon et le ballon s'élève dans les airs, mais s'il n'est pas soufflé... $»^{21}$. Il analyse ce succès en termes de «ballon», c'est-àdire, de phénomène passager et superficiel artificiellement créé ; «... M. Sartre, écrit Langevin, a donné une conférence au Windsor. Il faut croire que la publicité a été formidable, car il y avait du monde... et des grandes dames. Tous les quartiers chics de la métropole étaient dignement représentés... ». Jean Ampleman ${ }^{22}$

19. Ibidem, p. 364 .

20. Lucien Parizeau avait rencontré Sartre à New York en 1945 et en 1946 par l'intermédiaire de Jean Benoit-Lévy dont Parizeau avait publié Les grandes missions du cinéma en 1945 (Entretiens téléphoniques avec Yvan Cloutier, 5 juillet 1983 et décembre 1987 et Entrevue avec Lucien Parizeau, éditeur, réalisée par Silvie Bernier le 15 août 1984 à Ottawa, [document photocopié], Département d'Érudes françaises, Université de Sherbrooke, pp. 5-6, 21-22). Parizeau devait signer un contrat d'édition pour Morts sans sépulture et Introduction aux écrits intimes de Baudelaire (voir : Lucette ROBERT, "Ce dont on parle». Revue populaire, vol. 39, no 5 (mai 1946), p. 9.) Ces titres sont annoncés «en préparation» dans le Vient de paraître, cahier de l'actualité littéraire publié par les Éditions Lucien Parizeau er distribué en mai 1946, p. 12.

21. André Langevin, «M. Jean-Paul Sartre et l'Existentialisme», Le Devoir, vol. 37, n' 58 , p. 10.

22. Jean Ampleman, «Entrevues avec Sartre et Magali», Notre Temps, 16 mars 1946, p. 5 : «Jean-Paul Sartre, le prophète de l'existentialisme, est venu parmi nous. Tout ce 
Pierrette Cousineau ${ }^{23}$ et Guy Sylvestre ${ }^{24}$ reprendront ce facteur mondainisation. L'engouement pour Sartre serait un effet de publicité et de mondainisation et comme ce milieu mondain a un accès privilégié aux quotidiens (en particulier les carnets mondains, les pages féminines et les cahiers littéraires) mondainisation et médias sont difficilement dissociables.

Que la conférence ait été organisée sous les auspices de la Société d'étude et de conférences, fut, je crois, le facteur déterminant dans cette mondainisation. Le très grand succès de Huis clos au Gésù à la fin janvier 1946 avait déclenché la curiosité pour ce grand écrivain et philosophe dont le nom et quelques œuvres n'étaient connus que par de très rares individus (Sylvestre, O'Leary, P. Baillargeon, Parizeau et, sans doute, R. Garneau). L'article du Time Magazine entraîna une réaction de recul, voire même d'attaque, contre cet engouement. La réaction partit du Devoir 25 qui publia des repiquages d'articles de journaux et revues françaises hostiles à Sartre ; les Compagnons renoncèrent à leur projet de monter la pièce Les Mouches pourtant annonçée, et Pierre Gélinas exprima dans Le Jour sa crainte que la conférence de Sartre à la S.E.C. ne soit annulée. Selon Madame Suzanne F. Langlois, membre du Comité d'organisation de la conférence de Sartre, «...c'est devant les réactions négatives au sein même du comité d'organisation

que nos salons mondains contiennent de belles dames et de beaux jeunes hommes est accouru au passage de cet écrivain, sujet de toutes les discussions présentes dans nos milieux dits intellectuels. »

23. Pierrette Cousinfau, "Sartre et les "Mouches inutiles" ", Le Quartier Latin, vol. 28, n" 38 (15 mars 1946), p. 3: “Bzz... Czz.. oiseau... Ch... Ch... Czz... Chapeau-oiseau. Rrr... rr ... plumes de paon sur le derrière dd... dd.. du chapeau. Hommage de la femme montréalaise, au distingué Jean-Paul Sartre. (...) Coiffé par cinq cents femmes de cinq cents chapeaux différents, Jean-Paul Sartre, l'illustre révolté, a paru découvert devant son auditoire habillé. Cinq cents femmes perchées sur leur culture et leurs talons ont pâli devant la nudité de l'orateur. Sartre, le sans-chapeau. »

24. Guy Sylvestre, «Toujours Sartre. La curiosité existentialiste fait un peu sourire, dit M. Sylvestre », Le Canada, Vol. 43, nº 291 (15 mars 1946), p. 3 : « La curiosité existentialiste est purement et simplement du snobisme !... L'existentialisme est une philosophie et c'est en tant que philosophie qu'il doit être jugé ; le reste n'est que mode passagère et s'évanouira dès que les grandes ceuvres existentialistes seront offertes au public ».

25. Selon le Dictionnaire pratique des auteurs québécois (Fides, 1976, p. 401), après avoir été "messager, André Langevin devient journaliste au Devoir (responsable de la secrion litréraire) "; d'après le Dittionnaire des œuvres littéraires du Québec, Tome III, p. 356 (Fides 1982), il est chroniqueur littéraire au Devoir de 1945 à 1947. Cette réaction fut-elle menée par Langevin ou par la direction du Devoir? 
que Mme Dupuy ${ }^{26}$ est allée demander conseil à Monseigneur Charbonneau qui lui a dit : "J'aime mieux le voir parler à la Société d'étude et de conférences que partout ailleurs." » 27

Cette société d'animation culturelle recevait une triple consécration de son affiliation à la faculté de Philosophie de l'Université de Montréal, de la «direction éclairée» du Père Marie-Ceslas Forest, doyen de la Faculté de philosophie de l'Université de Montréal de 1926 à 1952 et secrétaire de l'Académie Canadienne St-Thomas d'Aquin, et de ses liens avec les ambassadeurs français ${ }^{28}$. Sartre ne pouvait avoir une meilleure tribune pour attirer public et journalistes comme en témoigne l'annonce parue dans La Presse du 9 mars : "Sous la présidence d'honneur de M. René de Messières, conseiller culturel près de l'ambassade de France à Ottawa... À la table présidentielle, on remarquera outre MM. de Messières et Sartre: la comtesse de Hautelocque, M. Robert Victor, consul général de France, et Mme Victor, le R.P. Ceslas Forest, o.p., Mme Pierre Dupuy,... M. et Mme Pierre Ricour ${ }^{29} \ldots{ }^{30}$.

L'institution organisatrice et le sujet de la causerie fournirent un visa idéologique à un double titre : une caution mondaine et une caution politico-littéraire grâce au titre de la conférence «La littérature française de 1914 à 1945 ; la littérature clandestine » qui attirera les tenants de la littérature «engagée » et les défenseurs de la France libre.

26. Mme Dupuy était l'épouse du diplomate et alors présidente de la S.E.C.; ce fut elle qui fit la présentation de Sartre et, selon Mme Langlois, ce fut elle qui invita Sartre à Montréal. Je n'ai pu contacter Mme Dupuy et Parizeau ne se souvient pas qui fut le responsable de la visite de Sartre à Montréal.

27. Lettre de Mme Suzanne Langlois à Y.C., 5 avril 1983 ; avant d'écrire cette lettre, elle a consulté Mme Maurice Hudon, alors responsable de la publicité. Dans une autre lettre du 10 mai 1983, Mme Langlois note que c'est à la suggestion du Père Ceslas Forest o.p., alors conseiller de la S.E.C., que Mgr Charbonneau fut consulté : «Le Père Forest n'avait pas d'objection à inviter Sartre mais, toujours sage et diplomate, il préférait s'en remettre à la décision de Mgr Charbonneau. »

28. "La Société d'étude et de conférences. Les choses intellectuelles plutôt que la broderie. " Perspectives, 25 mars 1978, p. 8.

29. Professeur au Collège Stanislas de Montréal.

30. La Presse, année 62, $\mathrm{n}^{\circ} 122$ (samedi le 9 mars 1946), p. 21. 


\section{LES DÉTERMINANTS DE CETTE RÉCEPTION DANS LES MÉDIAS}

Ainsi deux facteurs concourent à assurer une diffusion de Sartre: des médias qui veulent faire la nouvelle et une société « mondaine » qui veut augmenter son prestige. Sartre vient donc à Montréal pour «usage médiatique ». Parizeau ${ }^{31}$ veut mousser la publicité des livres de Sartre qu'il entend publier et élargir son public-clientèle et l'horizon d'attente de ce public. Un autre groupe, la nouvelle critique, va exploiter « médiatiquement » Sartre ; ce groupe a accrû son pouvoir avec la guerre sous la double impulsion d'une édition en très forte croissance et d'une ouverture médiatique des Québécois à ce qui se passe ailleurs depuis la guerre. Ces critiques vont exploiter la présence de Sartre pour renforcer leur position dans le champ intellectuel tant par rapport au courant traditionnel de la critique que par rapport à l'institution universitaire.

Les enjeux de ces stratégies médiatiques sont aussi politiques. Sartre sera moussé par le libéral Canada alors que la riposte viendra du Devoir ${ }^{32}$; les autres journaux et revues prendront position pour ou contre, les « pour » l'emportant grâce à l'action des journalistes et critiques.

Sartre représente pour ces derniers une valeur sûre pour trois raisons: 1) il répond aux critères qui déterminent l'horizon d'attente de l'époque tout en constituant un moyen d'infléchir l'horizon selon leur conception (Sartre s'inscrit dans un continuum et non en rupture ou en discontinuité comme ce sera le cas pour Borduas) ; 2) la position de Sartre dans le champ littéraire et dans le champ philosophique lui confère le statut d' «intellectuel total» d'autant plus que la «traductibilité » (philosophie-littérature) de son cuvre lui ouvre un marché virtuellement universel; 3) la thématique sartrienne explicite (dit «tout haut » ce que plusieurs pensent «tout bas») dans un discours articulé des thèmes qui se développent au Québec (liberté, authenticité, engagement de l'écrivain, etc.).

31. Qui a travaillé à L'agence canadienne de publicité avant de devenir éditeur.

32. Le Devoir redevint plus conservateur sous la fin du règne de Georges Pelletier et après le départ de Roger Duhamel. 


\subsection{Le pouvoir de la critique}

Pendant l'évolution tranquille de la fin des années trente, les revues culturelles se multiplient très rapidement ${ }^{33}$ et favorisent le développement d'une nouvelle couche d'intellectuels dont le pouvoir redoublera brusquement au cours des années de guerre. En quelques années, des barrières culturelles s'effondrent; la curiosité des Québécois pour les événements qui se déroulent à l'étranger est alimentée par la presse écrite et la radiophonie ; Montréal devient un centre nerveux de la culture française, on y reçoit de nombreux intellectuels français et on y édite et réédite beaucoup de livres.

L'édition subit une croissance qu'elle ne sera pas en mesure de digérer; alors que l'on édite 23 récits (romans, contes, nouvelles - grand public et jeunesse) en 1940, on en éditera 40 en 1942, 43 en 1943, 41 en 1944, 40 en 1945, 47 en 1946, 43 en 1947, 32 en 1948 et 15 en $1949^{34}$. L'élargissement du marché international fait des éditeurs des entrepreneurs qui cherchent à développer le marché local en multipliant les points de vente et en faisant de la publicité dans les journaux. En échange de publicité plusieurs journaux s'engagèrent à établir des pages littéraires ${ }^{35}$, d'où l'essor rapide de la critique littéraire. Ces éditeurs acquièrent rapidement un prestige et «vont disposer d'un certain pouvoir de consécration ${ }^{36}$ qu'ils vont exercer en usant de critères étrangers, surtout français.

33. Nouveaux périodiques répertoriés d'après Jacques BEAUDRY, Répertoire Revues littéraires québécoises $X I X^{e}$ et $X X^{e}$, [s.l., s.d., s.p.], (document photocopié) : 1932 (1), 1933 (1), 1934 (3), 1935 (5), 1936 (6), 1937 (3), $1938(0), 1939(1), 1940(3), 1941$ (5), $1942(0), 1943(1), 1944(1), 1945(1), 1946(3)$

34. Voir: Jacques MiCHON, "Croissance et crise de l'édition litréraire au Québec (1940-1959)», Littérature, no 66 (mai 1987), p. 115-126.

35. Ce qui est confirmé par le Publisbers Weekly du 14 septembre $1946:$ Early in the war period, the young publishers approached the various French newspapers in Canada, offering to supply them with ads if they would establish literary pages. Most of the papers took up the idea with enthousiasm. Every publisher, new and old, placed advertising. - Le Droit in Otcawa started a page in 1940, under Guy Sylvestre ; - Le Devoir in Montreal a page in 1942, under Roger Duhamel, who now does a similar page in the Montreal La Patrie [1946]. - Le Canada, Montreal, has had a big 20 page annual book supplement every fall, in 1943,1944, and 1945, and is probably going to do the same this fall. René Garneau supervises Le Canada's book section. - Le Petit Journal and La Presse, both in Montreal, also had book pages, as do L'Événement and Le Soleil in Quebec. ( (p. 1348). Je dois cette information a Jacques Michon.

36. Jacques MiCHON, “ L'édition littéraire au Québec, 1940-1960 », dans L'Édition littéraire au Québec de 1940 à 1960, [en collaboration], Sherbrooke, Département d'études 
Parizeau agit en éditeur en étant déjà informé de l'œuvre de Sartre, en ayant ses contacts avec les Français à New York, en servant d'impresario pour les écrivains ; il publie dans un journal le texte d'une conférence dans laquelle il était largement question de Sartre et il publie dans son cahier Vient de paraître ${ }^{37}$ un inédit de Sartre «Le dandysme de Baudelaire». Ce même Parizeau servira d'impresario à Camus en mai $1946^{38}$.

Revenons au développement de la critique littéraire. La critique recevra de l'édition la demande et elle exercera son pouvoir de consécration avec des critères semblables ${ }^{39}$. Les critiques ne se limiteront pas à suivre l'évolution des idées, ils deviendront des agents «en essayant de jouer un rôle dans l'orientation des esprits ${ }^{40}$. Comme le notent les auteurs du Dictionnaire des ouvres littéraires du Québec, «L'une des principales caractéristiques de la critique littéraire dans la presse quotidienne et périodique c'est qu'elle tente de rejoindre, et par le fait même, de former le goût du public pour une littérature nationale ${ }^{41}$. Le peu de recherche en littérature et l'absence d'enseignement de la littérature québécoise contribueront à fonder leur pouvoir de substitut dans le champ intellectuel.

La critique augmente son pouvoir lorsqu'elle se constitue en un réseau dans un champ culturel restreint; l'accentuation des différences y est moindre et la mobilisation potentielle du réseau fournit un levier important qui peut faire réfléchir tout opposant.

françaises, Université de Sherbrooke (Cahiers d'études litréraires et culturelles, n 9), 1985 , p. 12. En page 13: “L'éditeur n'est pas seulement un homme de culture, mais aussi un homme d'affaires dont la force instituante repose autant sur son capital symbolique que sur sa capacité commerciale. »

37. Op. cit., p. 10-11.

38. Des étudianrs de l'Université Laval ayant menacé de déclencher une protestation lors de cette conférence, Parizeau en abandonna le projet. Selon Parizeau, ces étudiants étaient «sans doute » ceux-là “qui chantaient les gloires de Pétain et les vertus de la collaboration ». Voir : Lucien Parizeau - Entrevue avec Claudette Lambert, dans la série “Mémoires ", diffusé à Radio-Canada (F.M.) le 26 août 1987 . Il faut se rappeler que la conférence de Sartre avait porté essentiellement sur Camus et que les positions anti-Pétain et anti-giraudistes de Sartre, Camus et Parizeau étaient connues.

39. Pour une description des critères, voir Hélène LAFRANCE, Yves Thériault et l'institution littéraire québécoise, Québec, I.Q.R.C., 1984, p. 58-60.

40. Dictionnaire des ceuvres littéraires au Québec, tome III, 1940 à 1959, sous la direction de Maurice Lemire, 1982, p. XLII

41. Ibidem, P. XL. 
Il serait simpliste de réduire la critique journalistique montréalaise à un seul réseau étanche; il se constitue néanmoins dans les années 40 un réseau informel important qui relie des individus appartenant à divers sous-groupes. Ces sous-groupes ont en commun de réunir des anciens des jésuites, par conséquent des individus formés à la pensée de Maritain et engagés dans la lutte pour la primauté du spirituel et pour le droit à une action autonome des laïcs. Ces individus, souvent groupés selon leur Alma Mater ${ }^{42}$, sont pour quelques-uns associés au Canada.

Deux intellectuels semblent exercer l'hégémonie, ce sont Robert Charbonneau et Roger Duhamel. Alors que la filière du premier est partisane de cet humanisme universaliste maritainien, la filière du second est nationaliste, regroupant entre autres des anciens Jeune-Canada ${ }^{43}$. J'inclus dans ce réseau: Élie, Hurtubise, Le Moyne, Saint-Denys Garneau, Frégault, Rex Desmarchais, O'Leary, Sylvestre, René Garneau, Marcel Raymond, Berthelot Brunet, André Laurendeau, Rodolphe Dubé, Jacques Lavigne, Baillargeon, Alfred Ayotte. N'avons-nous pas là les critiques des principaux journaux montréalais et les collaborateurs de plusieurs revues importantes? Et si nous ajoutons les amis des amis, nous sommes devant un groupe d'individus qui occupent des positions stratégiques dans le champ culturel québécois.

\subsection{Guy Sylvestre}

Les journalistes diffuseurs de Sartre, Dostaler O'Leary ${ }^{44}$, Alfred Ayotte ${ }^{45}$, Guy Sylvestre et Roger Duhamel ${ }^{46}$ ont tous rencontré Sartre lors de son premier voyage au Québec en mars 1945 , et ils gravitent autour de Guy Sylvestre qui a l'avantage

42. La Relève et La Nouvelle Relève regroupent des anciens de Sainte-Marie alors qu'Amérique française réunit des anciens de Brébœuf. De nombreux individus participent aux deux revues.

43. Duhamel et Charbonneau ont tous deux participé aux Jeunes-Canada ; Charbonneau a cependant modifié son nationalisme sous l'influence de Maritain.

44. Journaliste à La Patrie O'Leary collabore avec Sylvestre à Notre Temps; il a une connaissance directe des Temps modernes et son Le roman canadien-français (1954) fait une large place à Sartre.

45. Ayotte est l'auteur de l'entrevue de Sartre publiée dans La Presse du 11 mars 1946.

46. Quant à Duhamel, ce critique prestigieux présente dans La Patrie du 15 mars 1946 un compte rendu du « lucide exposé» de Guy Sylvestre au Cercle Universitaire. 
certain sur les autres d'avoir fait des études de philosophie ${ }^{47}$. Entre 1946 et 1952, Sylvestre écrira plus de douze textes autour de Sartre dont plusieurs témoignent d'une connaissance précise de la pensée sartrienne.

Sans l'action de Guy Sylvestre, la réception de Sartre aurait sans doute été tout autre; ce jeune anthologiste et critique conférera à Sartre une légitimité. Guy Sylvestre, qui n'a que 28 ans en 1946, a su cumuler un capital symbolique de son identification à un catholicisme du «oui », de sa consécration par le réseau, de sa stratégie visant à lui assurer une présence dans plusieurs milieux, de sa formation philosophique et de son appartenance au champ politique.

Partisan d'un "catholicisme du oui», Sylvestre déplore le «refroidissement général du catholicisme moderne» qu’il juge "trop négatif... peureux " ${ }^{48}$. C'est au nom d'un catholicisme ouvert qu'il défend Mauriac et qu'il voit dans l'apparition des philosophies existentielles le signe de l'avènement d'un catholicisme positif ${ }^{49}$. Cependant ce catholicisme «ouvert» n'est pas un catholicisme «mou ».

Maritainien ${ }^{50}$ par son exigence de l'ordre, de la distinction et du concret, Sylvestre ne sera pas ce «redresseur» rigide que fut Maritain et qui demeura, somme toute, un thomiste intransigeant comme en témoigne sa critique des philosophies de l'existence dans son Court traité de l'existence et de l'existant ${ }^{51 .}$

47. Dans le cadre du cours du Père Trudel, il avait travaillé à une bibliographie sur les principaux uuvrages er arricles contenus dans les bibliothèques d'Ottawa et portant sur les philosophies existenrielles, bibliographie dans laquelle il y a deux renvois à Sartre. Philosophies existentielles - Essai de bibliographie des principaux ouvrages et articles contenus dans les bibliothèques d'Ottawa, [date manuscrite 1940-41,22 p., s.l., s.é. |, index des noms cirés.

48. Guy Sylvestre, "Catholicisme. Pages de journal», Amérique française, vol. 1, no 4 (mars 1942), p. 39-44.

49. Ibid.

50. Comme en témoignent les nombreuses cirations d'appui dans les textes d'avant 1940 , l'« Hommage Maritain» qu'il publiera dans La Rotonde, vol. 8, no 9 (25 avril 1940), p. 4-5. Dans "Le don d'écouter chez Maritain", Écrits du Canada français, no 49 (1943), p. 88-114, Sylvestre écrit que le « rôle de Maritain fut... capital " pour lui dans sa formation philosophique, «écartelé [qu'il était] entre le foisonnement de Claudel et la rigueur de la Summa theologica", l'ouverture aux préoccupations temporelles et esthétiques fut aussi déterminante.

51. Paris, Paul Hartman Éditeur, 1947, 239 p. 
Comment être suspect devant quelqu'un qui dirige une revue, Gants $d u$ ciel $^{52}$, éditée par Fides? Le même éditeur publie son Poètes catholiques de la France contemporaine en 1943. N'est-il pas pris à partie à deux reprises par Pierre Gélinas qui le classe parmi les produits de «l'école néo-catholique, ceux qui ont suivi Maritain et Massis ${ }^{53}$ ?

Ce jeune critique est rapidement reconnu par ses pairs comme un des trois grands de la critique. Selon Gilles Marcotte, «[a]ux environs de 1945, ils [René Garneau, Roger Duhamel et Guy Sylvestre] exercent déjà le métier de critique depuis quelques années, et ils font autorité dans le milieu littéraire canadienfrançais ${ }^{54}$; ils partagent une solide connaissance des classiques et de la littérature française contemporaine, un souci de la langue écrite et un humanisme universaliste. En 1948, Jean Pierre Houle classe dans les grands de l'essai et de la critique: R. Garneau, R. Duhamel, L.-M. Raymond, Guy Frégault ${ }^{55}$ et Guy Sylvestre ${ }^{56}$. En 1951, Duhamel ${ }^{57}$ place Sylvestre parmi les trois plus grands de la critique au Québec, après «le prince de la critique au Canada français », René Garneau, et avant Marcel Raymond.

52. De septembre 1943 à l'été 1946. Sylvestre avait soumis le projet à Fides dont le rôle se limita au support logistique: comptabilité, stockage et distribution. Voir: Guy SYLVESTRE, “Gants du Ciel », dans Revue d'bistoire littéraire du Québec et du Canada français, no 6 (été-automne 1983), p. 65-67.

53. Pierre GÉlunas, compte rendu de Sondages dans la rubrique « Chronique des livres » du Jour, vol $8, n^{0} 29$ (24 mars 1945), p. 5. Gélinas avait jugé très sévèrement le premier numéro de Gants $d u$ Ciel; il y avait accusé Sylvestre d'être de "droite ", de ne pas avoir créé une revue où les considérations de l'art seul primerait, et il reprochait à cet «internationalisme de surface de ne pas favoriser les créateurs québécois.

54. Gilles MarcotTe, “La critique des journaux et des revues» dans Histoire de la littérature française du Québec, tome IV. Roman, théâtre... (de 1945 à nos jours), (sous la direction de Pierre de Grandpré), Montréal, Beauchemin, 1969, p. 341.

55. Guy SYIVESTRE fait la présentation de Guy Frégault dans « Voici notre jeune littérature 》, Revue populaire, vol. 39, no 10 (octobre 1946), p. 12 et 73 . Il se permer de le critiquer et par là se pose en position d'autorité par rapport à Frégault.

56. Dans : «Une enquête de Jean-Marc Léger - Où va la littérature canadienne-française? - La réponse de M. Jean-Pierre Houle», Le Devoir, vol. 39, no 130 (5 juin 1948), p. 11. Récemment, Paul Beaulieu rappelait l'autorité de ce trio: «mais comment récuser les jugements d'analystes dont l'autorité est bien assise, tels René Garneau, Roger Duhamel, Guy Sylvestre »; dans : " Robert Charbonneau : esquisse d'un portrait », Écrits du Canada français, no 57 (1986), p. 22.

57. Roger Duhamel, « La critique et le critique », La Nouvelle Revue Canadienne, vol. 1, $\mathrm{n}^{\circ} 2$ (avril-mai 1951), p. 23-24. 
Comme Sartre en France, Sylvestre parvint à occuper dans le champ intellectuel québécois ${ }^{58}$ une place de premier plan grâce à une stratégie très efficace où sont mis à contribution divers procédés tels demandes de préface, dédicaces de ses textes, citations et références d'appui, annonces de publications ${ }^{59}$, diversification dans le choix des lieux de publication ${ }^{60}$ et correspondance.

Sylvestre, surtout si on considère qu'il devient fonctionnaire dès la fin de ses études, fut un critique et essayiste des plus prolifiques. En plus d'alimenter sa propre chronique littéraire au Droit, il collabora à l'époque à plus de 19 revues différentes, sans compter les nombreux journaux; il publie pas moins de 106 comptes rendus dans Notre Temps entre novembre 1945 et octobre $1951^{61}$.

En plus d'une légitimité qui reposa sur une base élargie, un des effets d'une telle stratégie fut de produire un Sylvestre " polymorphe » qui ne saurait être réduit à l'étiquette d'une maison d'édition ou d'une revue, d'un mouvement ou d'un courant d'idées - bref il ne pourra devenir une bonne cible pour ses opposants.

Sylvestre tire aussi une légitimité de sa formation philosophique. Il réfère à Aristote, Kant, Spinoza et Maritain dans des propos sur les rapports entre poésie et métaphysique ${ }^{62}$; il disserte sur des questions d'esthétique comme le rapport de la forme et du contenu, connaissance poétique et connaissance philosophique, etc. ${ }^{63}$; il

58. Je me limite ici au champ québécois mais le champ-cible de Sylvestre étair le champ canadien-français, voire même canadien, comme en témoignent de nombreuses publications en langue anglaise et quelques-unes bilingues. Ceci n'est pas sans lui conférer quelque autorité.

59. Il annonce dans ses livres les publications en préparation, ce qui nous informe des orientations idéologiques, et donne aussi l'impression d'une plus grande productivité.

60. La diversité des lieux de publication est remarquable: de L'Action nationale à La Nouvelle Revue Canadienne (Ottawa) en passant par Regards (Québec) aux Carnets Viatoriens (Jolietre), Horizons (Trois-Rivières), sans oublier les revues universitaires, telles L'Action Universitaire et celles d'Ottawa et de Québec ; donc diversité géographique et idéologique.

61. Il faut tourefois prendre en considération les reprises. Il ne s'agit pas d'un « repiquage », mais de plusieurs textes à partir d'un même contenu dans des revues différentes, ce qui lui assure une plus grande diffusion.

62. Voir sa lettre au Père Gustave Lamarche publiée dans Les Carnets Viatoriens, Ge année, $n^{\circ} 4$ (octobre 1941).

63. Dans l'introduction à son Antbologie 1943 ; voir "Qu'est-ce que l'art?» dans Sondages. 
manie aisément les distinctions. Cette autorité du philosophe en fera un critique crédible de l'existentialisme d'autant plus qu'il est présenté par Duhamel comme une compétence dans le domaine:

Sa solide formation philosophique lui confère quelque autorité pour étudier en de subtiles analyses les courants les plus confus de la pensée de notre temps. Qu'il traite de l'existentialisme ou qu'il décortique les éléments de l'humanisme chrétien repensé par Maritain, il y apporte toujours la même maturité intellectuelle, le même souci d'impartialité qui n'est pas froideur, mais respect pour les plus hautes projections de la pensée humaine. Sylvestre est notre Thibaudet - et sous ma plume ce rapprochement est plus qu'un éloge banal. ${ }^{64}$

Enfin un travail de fonctionnaire assurera à Sylvestre une autonomie financière et intellectuelle; il ne sera pas à la merci des directions de journaux et des pressions de la vie politique québécoise. La fonction publique et Radio-Canada ont constitué une espèce d'enclave dans le Québec duplessiste ; les individus s'y sentaient dotés d'une sorte d'immunité. Il travaillera d'abord comme traducteur au Secrétariat d'État (1942-44), il sera ensuite attaché d'édition à la Commission d'information en temps de guerre (1944-45), et il devient secrétaire particulier du Secrétaire d'État aux Affaires extérieures, qui était alors Louis Saint-Laurent, pour enfin occuper le poste de secrétaire particulier (1948-50) de ce dernier, devenu Premier Ministre.

Les fonctions politiques de Sylvestre auprès de Saint-Laurent vont lui conférer une autorité certaine; Duhamel ne mentionnet-il pas la fonction politique du conférencier Sylvestre dans sa présentation au Club universitaire? Par contre, ces charges vont exiger de Sylvestre une position de distanciation dans le champ intellectuel.

De plus, le travail de Sylvestre à Ottawa le mettait en contact avec le milieu diplomatique, en particulier le milieu diplomatique français d'Ottawa (que les dominicains fréquentaient eux aussi). Ne pas oublier que ces diplomates étaient en mission au Canada!

Voilà le réseau-support du sartrisme et son principal «illustrateur » Guy Sylvestre. Après avoir identifié les bases institutionnelles et les positions idéologiques du récepteur, nous sommes

64. "Guy Sylvestre, par Roger Duhamel », dans la rubrique «Voici notre jeune littérature ", Revue Populaire, vol. 39, no 10 (octobre 1946), p. 12 et 73. 
en mesure d'évaluer en quoi Sartre peut servir les intérêts de ce groupe et des « illustrateurs » tels Sylvestre et O'Leary ; en d'autres termes il s'agit d'évaluer le capital symbolique de Sartre.

\subsection{Sartre: un capital symbolique}

La critique trouvera en Sartre un objet qui répond à ses attentes et qui peut lui permettre d'accroître son pouvoir dans le champ philosophico-littéraire; considérons brièvement les trois raisons de cet intérêt que nous avons énoncées au début de cette section.

\subsubsection{L'horizon d'attente}

Sartre répond très bien aux critères qui déterminent l'horizon d'attente de la période 1944 à 1964 . Selon Hélène Lafrance ${ }^{65}$, ces critères sont les suivants : référence à des modèles français (1), la maîtrise de la langue française (2), l'originalité et le talent (3), l'universalité (4), la vraisemblance du récit (5), la spiritualité (6), la moralité (7), et la méfiance envers la littérature «populaire» (8). Ces critères sont partagés par les deux grands courants de la critique, celui (rattaché au pouvoir du clergé et à l'appareil scolaire) que l'on identifie à l'idéologie de conservation et l'autre groupe relativement hétérogène et que l'on identifie à l'idéologie de rattrapage.

Ces courants diffèrent et s'opposent dans leurs interprétations et la pondération de ces critères. Les deux groupes s'opposent quant à l'importance relative du critère moral; alors que l'idéologie de conservation accorde une priorité à la lecture morale sur les qualités esthétiques, les promoteurs de l'idéologie de rattrapage, selon Lafrance, «jugent... les œuvres selon des critères presque purement esthétiques et selon la dimension d'universalité qu'elles contiennent... Pour eux, les critères moraux n'ont plus une importance vitale, mais des préoccupations spirituelles comme celles qu'on retrouve chez les romanciers chrétiens français demeurent

65. LAFRANCE, op. cit., p. 58-59. 
nécessaires » ${ }^{66}$. Tout comme Daniel-Rops ${ }^{67}$, certains Québécois font une analyse symptômale et récupératrice de la popularité de Sartre ${ }^{68}$.

La critique traditionnelle est prise au dépourvu certes à cause de son ignorance de l'œuvre de Sartre, mais un tel engouement des catholiques face aux premières œuvres de Sartre n'est pas propre au Québec. Selon Beigbeder, la réception positive par le milieu chrétien est une des raisons importantes du succès de Huis clos en France; ces chrétiens «persistaient, malgré la position négatrice de l'auteur, à y trouver l'écho d'une inquiétude religieuse ${ }^{69}$. Un chrétien n'est-il pas réceptif devant une œuvre qui recourt à l'imagerie religieuse et qui reprend la thématique bernanosienne de l'enfer et du regard ${ }^{70}$; la question de l'engagement est à l'ordre du jour des jeunes lecteurs d'Esprit et, bien avant, dans ce christianisme militant des Bloy et des Péguy. Selon Galster ${ }^{71}$, Marcel fut l'un des médiateurs principaux de l'œuvre sartrienne avec une jeune critique catholique sympathique à Sartre, Claude-Edmonde Magny ${ }^{72}$.

Nos catholiques partagent cette première ouverture mais ils divergeront dans leurs attitudes face à Sartre: attaque frontale, récupération et dialogue. La réaction, amorcée dans Le Devoir, se fera par repiquages d'articles de journaux et de revues françaises

66. Ibidem, P. 59-60

67. Daniel-Rops, “Littérature d'un monde en perdition ", La Nouvelle Relève, vol. IV, no 9 (mars 1946), p. 18.

68. «La critique digne de ce nom doit prêter l'oreille aux intentions profondes qui orientent une cuvre, il doit savoir discerner les forces qui la travaillent en son épaisseur. À ce moment de l'interprétation, il répond à une injonction intime de l'auvre, mais il dépasse le plan esthétique », R.P. Pierre ANGers, s.j., “ La critique littéraire ", Association canadienne des bibliotbécaires de langue française. Texte des communications présentées au neuvième Congrès annuel tenu à Montréal, du 10 au 12 octobre 1953, Montréal, Bibliothèque de l'Université de Montréal, 1953, p. 113. Sous le pseudonyme F.R., Robert Charbonneau a écrit à propos de Huis clos que la pièce "n'en déplaise à son auteur, aurait pu être écrite par un catholique", dans "L'athéisme au théâtre", La Nouvelle Relève, vol. IV, n 8 (février 1946), p. 730.

69. Marc Beigbeder, L'bomme Sartre, Paris, Bordas, 1947, p. 33-34.

70. Voir : Ingrid GALSTER, Le théâtre de Jean-Paul Sartre devant ses premiers critiques, Tübingen-Paris, Gunter Narr Verlag - Jean-Michel Place, 1986, p. 295 sq. et Brian T. FiTCH, «Bernanos précurseur de Sartre : aspects sartriens de la dialectique du regard dans l'univers bernanosien », dans : Études bernanosiennes - 6 Confrontations, La Revue des Lettres Modernes, nos 127-129, 1965, p. 25-41.

71. Idem., p. 302.

72. Elle collabore tant au Monde qu'à Esprit et à Poésie 46. 
sauf pour quelques attaques virulentes caractérisées par l'ignorance de l'œuvre sartrienne ${ }^{73}$. Pour un, André Langevin passera de l'engouement du 28 janvier, au recul du 2 février, à la reconnaissance de son "brouillard existentialiste» le 11 mars, pour aboutir en septembre à une dénonciation des romans de Sartre comme avilissants pour la jeunesse et à la justification de la prohibition de l'œuvre ${ }^{74}$. Étonnant qu'on ait fait de Langevin un petit Sartre québécois à partir des années 60 !

Quand la critique traditionnelle intervient, les traînées médiatiques positives sont déjà là. L'attitude générale de ce groupe sera le flottement; il ne faut pas oublier que la philosophie sartrienne ne fut soumise par le Pape à l'examen de l'Académie pontificale de philosophie qu'en avril 1947 et ne sera condamnée que le 27 octobre $1948^{75}$.

\subsubsection{L'intellectuel total}

Boschetti montre bien comment Sartre va entreprendre de devenir l' « intellectuel total » en France. « Tout le champ intellectuel est impliqué, écrit-elle, Sartre s'impose à l'attention du monde philosophique et du monde littéraire, au monde de la culture noble et à celui des grands quotidiens, et il franchit ainsi les deux barrières - qui caractérisent le fonctionnement du champ pendant toute son histoire - entre circuit philosophique et circuit littéraire, entre succès légitime et divulgation. " ${ }^{76}$

La polémique autour de Sartre témoigne d'une nette dissociation entre ces deux champs. La philosophie a occupé la fonction de légitimation des autres discours et c'est la raison pour laquelle l'Église en a fait un champ clos qu'elle pouvait contrôler. La philosophie appartient au philosophe; dans leur recul, Béraud et Langevin se cantonnent dans le champ littéraire et Langevin regrette même sa malencontreuse aventure philosophique.

73. Deux exemples de critiques virulents : Marc AUBRY, «La querelle existentialiste», Revue Dominicaine, vol. 52, Tome 1 (février 1946), p. 109-112. Jacques Mathieu, «Littérature dissolvante », L'Action universitaire, vol. 12, n० 7 (mars 1946), p. 9-11.

74. André Langevin, « Un grand roman français », Le Devoir, vol. 37, n² 218 (21 septembre 1946), p. 8.

75. G.E., "The Works of Sartre Condemned», Clergy Review, 1949, pp. 59-60.

76. Anna BosCHETTI, op. cit., p. 18. 
La légitimité rattachée à la philosophie exerça une attraction sur les partisans d'un changement dans le champ intellectuel; ils avaient fait un premier apprentissage philosophique dans les deux dernières années du cours classique. René Garneau avait fait des études philosophiques à Paris tout comme André Laurendeau; nous savons que Robert Charbonneau aurait préféré devenir philosophe ${ }^{77}$ et que Guy Sylvestre a complété ses études de philosophie.

Sartre a l'intérêt de diffuser une philosophie dont le public virtuel soit universel à cause de la « traductibilité » du philosophique et du littéraire de sorte que le spectateur d'une pièce de Sartre se sent tout aussi justifié de parler de l'existentialisme que le lecteur de L'être et le néant. Le problème pour le censeur ne réside pas dans les ouvres philosophiques peu accessibles et «réfutables» mais dans les œuvres littéraires qui laissent des traces indélébiles ; voilà pourquoi on ne trouvait pas d'œuvre littéraire de Sartre à la bibliothèque de l'Université de Montréal en $1950^{78}$ alors qu'on y trouvait ses œuvres philosophiques. Autre fait révélateur, un des premiers philosophes à parler de Sartre dans son cours d'histoire de la philosophie, Jacques Lavigne, se servait de La nausée ${ }^{79}$, dont il avait obtenu une copie par un littéraire, Pierre Baillargeon ${ }^{80}$. Même l'Abbé Jean Milet ${ }^{81}$, titulaire du premier cours consacré à Sartre en 1951-52, «fait une étude assez poussée du texte de la NAUSÉE, qui était paru sept ou huit ans plus tôt et qui faisait encore "sensation"... » ${ }^{82}$. Le littéraire précède ici le philosophique ; en outre, Milet attribuera à la très grande popularité de Sartre à Montréal le fait que le doyen Forest lui ait demandé de donner un cours sur Sartre ${ }^{83}$.

77. Voir: Ducrocq-Polrier, op. cit., p. 33. Il fit en 1934 une causerie radiophonique intitulée "L'esprit de la philosophie française» à C.K.A.C.; selon Roger Larue, Robert Charbonneau - Bio-bibliograpbie, Montréal, [École de Bibliothéconomie], p. 17.

78. Dans Le Quartier Latin, vol. 32, n' 30 (14 février 1950), p. 1, Pierre Perreault signe un article intitulé "La famine menace les rats de bibliothèque»: "De Sartre, toute la philosophie, tout ce qui est illisible, mais aucun roman ni aucune pièce de théâtre."

79. Ce que confirment les notes de cours de l'étudiant Roland Houde [document manuscrit, 42 pages 1 .

80. Lettre de Jacques Lavigne à Y.C. (27 juin 1983).

81. Professeur à Stanislas.

82. Lettre de Jean Milet à Y.C. (2 octobre 1984).

83. Ibidem. 


\subsubsection{Sens commun et philosophe}

Sartre a un succès parce qu'une machine « médiatique » extraordinaire a bien fonctionné ; mais si cette machine a été mise en marche c'est parce que les promoteurs croyaient aux contenus à transmettre. Ainsi Lucien Parizeau, qui fut un ardent défenseur de la liberté dans Le Canada, L'Ordre et dans sa chronique à CKAC, admirait la notion sartrienne de liberté; une telle philosophie ne pouvait que servir à toute une génération en quête de liberté personnelle ${ }^{84}$. De même pour Sylvestre, l'existentialisme a une «portée symptomatique», ses succès «s'expliquent encore par le besoin que toute société ressent fortement de découvrir de nouvelles valeurs à la suite d'un profond bouleversement social... ${ }^{85}$. D'autres ancrages idéologiques sont identifiés dans les textes: le pourautrui, l'authenticité, l'engagement, la fonction de l'écrivain, l'athéisme, etc., de sorte que chacun avait presque son petit Sartre pour usage personnel. Cette «traductibilité » entre expérience vécue et philosophie rend la philosophie «organique » au sens gramscien du terme, c'est-à-dire, «catharsis d'une vie pratique donnée ${ }^{86}$, et c'est ce qui rend possible sa diffusion et surtout sa pénétration. Une philosophie devient réelle quand elle dit «tout haut " et d'une manière articulée et réfléchie ce que les gens pensent « tout bas» confusément.

\section{CONCLUSION}

Une société en transition, des groupes qui luttent pour une plus grande liberté, des journalistes et critiques qui trouvent chez Sartre une philosophie qui traduit leur préoccupation; voilà les composantes du phénomène Sartre qui deviendra médiatique par la prise en charge d'une philosophie par des individus occupant

84. Voir: Entrevue avec Lucien Parizeau, éditeur, réalisée par Silvie Bernier, Sherbrooke, Département d'études françaises, le 15 août 1984, [document photocopié], p. 8 et 20.

85. Guy SyLvestre, "L'Existentialisme est-il un humanisme?», Notre Temps, vol. 1, nos $42-43$ (10 août 1946), p. 4.

86. Voir : Yvan Cloutier, "Gramsci et la question de l'idéologie», Pbilosophiques, vol. X, no 2 (octobre 1983), p. 252. 
des positions stratégiques dans le champ intellectuel et qui voient des ancrages de cette philosophie dans les attentes de nombreux Québécois.

Mai 1986 et septembre 1987

Département de Pbilosopbie,

Collège de Sherbrooke

et Université de Sherbrooke. 